\title{
artigo
}

\section{Interfaces da terapia ocupacional em um serviço de atenção primária de Belém: relato de experiência}

\author{
Occupational therapy interfaces in a primary care service in Belém: experience report
}

Interfaces de terapia ocupacional en un servicio de atención primaria de Belém: informe de experiencia

\section{RESUMO}

O objetivo desse artigo é apresentar interfaces da Terapia Ocupacional na Atenção Primária, através do relato da experiência de um terapeuta ocupacional residente e seus preceptores. Trata-se de um relato de experiência descritivo de abordagem qualitativa, gerado a partir das vivências em uma Unidade Municipal de Saúde de um bairro periférico da cidade de Belém. O residente e preceptoras adotavam diferentes abordagens, de acordo com a necessidade de saúde dos usuários do serviço, atuando através de visitas domiciliares, grupos terapêuticos e atendimentos em vigilância do desenvolvimento infantil. Observou-se corroboração das atividades dos terapeutas com a literatura pesquisada, demonstrando que Terapeutas Ocupacionais apresentam atuação validada na Atenção Primária brasileira.

DESCRITORES: Atenção Primária à Saúde; Terapia Ocupacional; Estratégia Saúde da Família; Áreas de Pobreza.

\section{ABSTRACT}

The purpose of this article is to present interfaces of Occupational Therapy in Primary Care, through the report of the experience of a resident occupational therapist and his preceptors. generated from the experiences in a Municipal Health Unit in a peripheral neighborhood of the city of Belém. The resident and preceptors adopted different approaches, according to the health needs of the service users, acting through home visits, therapeutic groups and care surveillance of child development. activities of therapists with the researched literature, demonstrating that Occupational Therapists present validated performance in Brazilian Primary Care.

DESCRIPTORS: Primary Health Care; Occupational Therapy; Family Health Strategy; Poverty Areas.

\section{RESUMEN}

El objetivo de este artículo es presentar interfaces de Terapia Ocupacional en Atención Primaria, a través del relato de la experiencia de un terapeuta ocupacional residente y sus preceptores. Este es un informe de una experiencia descriptiva con un enfoque cualitativo, generado a partir de las experiencias en una Unidad Municipal de Salud en un barrio periférico de la ciudad de Belém. El residente y los preceptores adoptaron diferentes enfoques, de acuerdo con las necesidades de salud de los usuarios del servicio, actuando a través de visitas domiciliarias, grupos terapéuticos y vigilancia del cuidado del desarrollo infantil. actividades de los terapeutas con la literatura investigada, demostrando que los terapeutas ocupacionales presentan desempeño validado en la atención primaria brasileña.

DESCRIPTORES: Atención Primaria de Salud; Terapia Ocupacional; Estrategia de Salud Familiar; Áreas de Pobreza.

RECEBIDO EM: 29/01/2021 APROVADO EM: 10/03/2021

\section{Carlos Roberto Monteiro de Vasconcelos Filho}

Terapeuta Ocupacional, Residente do Programa de Residência Multiprofissional da Universidade do Estado do Pará.

ORCID: 0000-0003-3456-7323

\section{Solange Rezende Rabelo de Lima}

Terapeuta Ocupacional da Secretaria Municipal de saúde, Mestre em Saúde na Amazônia, formada pela Universidade do Estado do Pará.

ORCID: 0000-0003-4179-3310 


\section{INTRODUÇÃO}

A Atenção Primária a Saúde (APS) pode se apresentar de diferentes formas, isso presume a possibilidade de diferentes definições para esse serviço. Um dos primeiros esforços mundiais para definir a APS foi a Declaração de Alma-Ata ${ }^{1}$, que traz consigo a importância de um serviço de saúde que seja próximo e atuante na comunidade, além de promover a saúde em um conceito ampliado, explorando ações intersetoriais, rompendo com formas retrogradas de se fazer cuidado em saúde. No Brasil, o Sistema Único de Saúde (SUS), prevê a organização legislativa e política da APS em um modelo ampliado, assemelhando-se com as características descritas pela Declaração de Alma-Ata ${ }^{1}$

Assim, a Atenção Primária brasileira segue uma estratégia de universalidade, integralidade e equidade, visando a saúde com um conceito ampliado e atuando nas necessidades do usuário. A APS também assume o papel de primeiro contato com o usuário, permitindo acesso e utilização do serviço para cada necessidade ou episódio de saúde ${ }^{2}$.

É no contexto da APS que se insere o Eixo Estratégia Saúde da Família do Programa de Residência Multiprofissional da Universidade do Estado do Pará. Este eixo tem foco em promover vivências de profissionais de Terapia Ocupacional, Fisioterapia, Odontologia e Enfermagem na atenção primária, atuando de forma interdisciplinar e integral na rede de saúde. Os Terapeutas Ocupacionais do programa atuam na assistência à saúde em Unidades Municipais de Saúde, Núcleos Ampliados de Saúde da Família e também na gestão municipal e estadual de saúde, trazendo para suas práticas, os conceitos da profissão.

Portanto, a Terapia Ocupacional é profissão que trabalha da prevenção ao tratamento de indivíduos com alterações sociais, cognitivas, afetivas, perceptivas e psicomotoras, de qualquer origem, através da utilização da atividade humana como base de desenvolvimento de projetos terapêuticos específicos. Os profissionais acreditam que o fazer e o engaja- mento em ocupações significativas pode ser meio e/ou fim para tratamentos de diferentes condiçôes ${ }^{3}$.

O Conselho Federal de Fisioterapia e Terapia Ocupacional, na resolução $N^{\circ}$ 407 de 18 de agosto de $2011^{4}$, reconhece a possibilidade e especialidade de atuação da Terapia Ocupacional na atenção primária, dentro do programa saúde da família, planejando, coordenando, desenvolvendo, prescrevendo, acompanhando e reavaliando estratégias de intervenção para promover saúde, independência e autonomia no cotidiano dos usuários.

Apesar da existência de resoluções que respaldam terapeutas ocupacionais na atenção primaria à saúde, os autores, a partir das vivências nesse contexto, têm o interesse em compartilhar as ações realizadas em um serviço da APS, bem como verificar se estas atuações são respaldadas pela literatura nacional, principalmente quando inseridos em um contexto de comunidades fragilizadas. Dessa forma, a pergunta desta pesquisa é: como terapeutas ocupacionais atuam em serviços de Atenção Primária?

Já o objetivo desse artigo é apresentar interfaces da Terapia Ocupacional na Atenção Primária, através do relato da experiência de um terapeuta ocupacional residente e suas preceptoras em uma Unidade Municipal de Saúde de um bairro periférico da cidade de Belém.

\section{MÉTODO}

Trata-se de um relato de experiência descritivo de abordagem qualitativa, gerado a partir das vivências de um Terapeuta Ocupacional no primeiro ano do Programa de Residência Multiprofissional da Universidade do Estado do Pará, no eixo Estratégia Saúde da Família e Atenção Primária, bem como duas Terapeutas Ocupacionais preceptoras do Programa. A Residência em questão tem o objetivo de promover a formação e especialização de profissionais da saúde, com foco na atuação na Atenção Primária, dentro das potencialidades do Sistema Único de Saúde.

A fim de organização didática dos da- dos, o trabalho foi construído através de quatro etapas: descrição das experiências enquanto terapeutas ocupacionais atuantes na atenção primária; levantamento bibliográfico; análise da literatura e corroborações com as práticas realizadas. As atividades ocorreram nos meses de outubro a dezembro, nos períodos da manhã e tarde, nos estabelecimentos de atenção primária do Bairro do Telégrafo na cidade de Belém, principalmente na Unidade Municipal de Saúde do Telégrafo (UMS Telégrafo), bem como outras Unidades de Estratégia Saúde da Família do bairro.

A busca de artigos e bibliografias foi realizada nos seguintes bancos de dados: Biblioteca Virtual em Saúde (BVS); SCIELO; e LILACS. Os descritores utilizados foram somente na língua portuguesa, por acreditar-se que o estudo procurava aproximar os achados na literatura à realidade das cidades brasileiras. Os descritores foram: Terapia Ocupacional; Atenção Primária de Saúde; Saúde da Família. Além disso, limitou-se a pesquisa para os anos de 2010 a 2020.

\section{RELATO DA EXPERIÊNCIA}

A atuação enquanto Terapeuta Ocupacional na Atenção Primária no bairro do Telégrafo, adotava diferentes perfis de acordo com os dias e horários das ações. Pela manhã, os atendimentos estavam focados na realização de grupos e também no território, através de Atendimentos Domiciliares em conjunto com a equipe do NASF e Agentes Comunitários de Saúde. Já pela tarde, a predominância era de acompanhamentos de vigilância do desenvolvimento e intervençôes ao público infantil, dentro da UMS do bairro.

Os grupos enfocavam na temática de saúde mental, utilizando recursos como rodas de conversa, relaxamentos e dinâmicas para promover bem-estar mental dos usuários e também da equipe multiprofissional. A proposta de um grupo de saúde mental surgiu devido a observação de demandas de sofrimento dos usuários, em questão do enfrentamento da pandemia, o isolamento social e também a perda de 


\section{artigo}

familiares, amigos e usuários da unidade devido a COVID-19.

Os Atendimentos Domiciliares ocorriam com o objetivo de levar a promoção da saúde para dentro das residências dos moradores do bairro, dessa forma, as demandas apresentadas pela população eram variadas, perpassando por: pacientes pós-AVE e pós traumas, demandas sociais e de saúde mental, reabilitação cognitiva e demandas familiares. Assim, os Terapeutas Ocupacionais se utilizavam de atividades e orientações visando permitir o engajamento, participação e funcionalidade desses usuários em suas ocupações significativas.

Com a finalidade de prover melhor atendimento durante as visitas domiciliares, permitindo uma melhor aquisição de dados nos aspectos pessoais e ocupacionais dos pacientes, foi elaborada um formulário de avaliação terapêutica ocupacional para ser utilizada com os usuários, observando suas demandas, dentro dos domicílios e realidades. Esse formulário é dividido em duas partes, sendo a primeira a aquisição de dados pessoais e a segunda a aplicação de protocolos validados para o território brasileiro. A primeira parte foi elaborada com base na "Ficha de Cadastro Domiciliar e Territorial"s utilizada pelos Agentes Comunitários para levantar e cadastrar dados dos usuários no e-SUS

\section{Figura 1: Grupo de relaxamento}

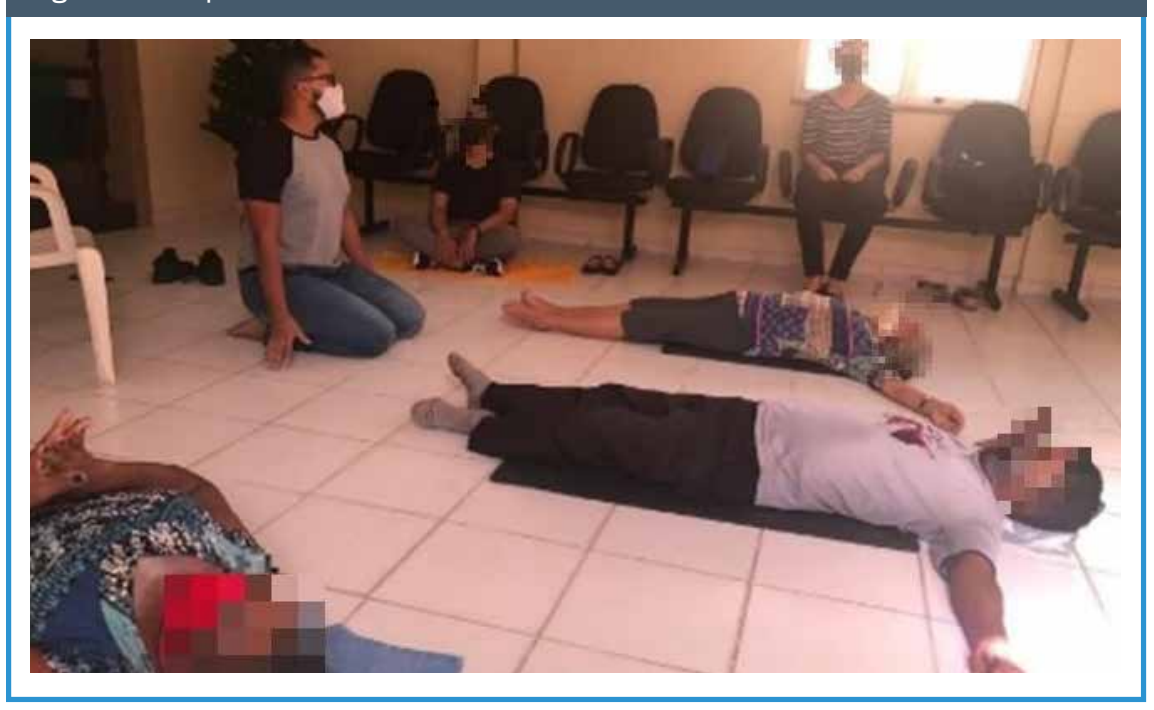

Fonte: Autores, 2020

Atenção Primária (e-SUS APS) que é um sistema de informações do SUS utilizado para sistematizar e digitalizar os dados de usuários adscritos em uma determinada área de abrangência. Já a segunda parte do formulário, se caracterizava pela utilização de avaliações amplamente utilizadas por Terapeutas Ocupacionais em sua prática, como o Mini Exame do Estado Mental, Escala de Barthel, Escala Geriátrica de Depressão, Índice de Katz e Medida de Independência Funcional. Esses instrumentos foram escolhidos devido as demandas percebidas pelos terapeutas e também pela afinidade dos mesmos na utilização.

Quanto as atividades que ocorriam no período vespertino, como já ditas anteriormente, estavam diretamente relacionadas com a vigilância do desenvolvimento, com objetivo de prevenir, acompanhar e promover saúde durante o processo de crescimento e desenvolvimento de crianças típicas e não típicas.

O serviço de vigilância foi instaurado após a percepção da terapeuta ocupacional da unidade, da necessidade de um programa que fornecesse assistência à saúde a esta população, sendo uma constante demanda dos usuários que buscavam auxilio dentro da Unidade. Os pacientes eram encaminhados ou acolhidos por demanda espontânea, sendo agendados para a realização de uma avaliação do desenvolvimento neurológico, motor, sensorial e também ocupacional. Após a avaliação, a terapeuta ocupacional, residente e o familiar entravam em acordo e decidiam quanto as demandas apresentadas, as necessidades a serem trabalhadas e também se o acompanhamento seria semanal ou mensal.

Além disso, se era prezado pela integralidade na atenção e também pelo trabalho em conjunto dentro da rede de serviços do município, dessa forma, em muitos casos, havia a necessidade de atendimento em outros níveis de atenção, como o secundário e terciário, com pacientes referenciados e contrareferenciados.

As ações, tanto pela manhã, quanto pela tarde, eram realizadas de forma conjunta entre preceptores e residente. Análises de casos, leitura de artigos, discussões com equipe e formulação de planos terapêuticos singulares faziam parte dos procedimentos entre preceptores e residente, além dos próprios atendimentos. O preceptor atuava como um orientador, auxiliando na tomada de decisões do residente, de acordo com as necessidades do contexto e do usuário a ser atendido.

A pandemia da COVID-19 trouxe a necessidade de alterações nos processos dos atendimentos. Durante a manhã, os grupos precisavam ser com público reduzido, respeitando o distanciamento social e também com menor tempo de exposição. As visitas também foram reduzidas, além de ocorrerem com equipes menores e com usuários fora do grupo de risco. Nas atividades da tarde, a quantidade de atendimentos foi reduzida, passando de 6 para 3 atendimentos diários, com intervalos de 10 a 15 minutos entre as sessões, a fim de higienizar o espaço e o material. Foi adotada a estratégia de hora marcada, reduzindo o tempo em que o usuário espera pelo atendimento, consequentemente, diminuindo sua exposição.

\section{DISCUSSÃO}

O bairro do Telégrafo está localizado na Bacia do Una, na região da baixada periférica de Belém ${ }^{6}$. O território é ma- 
joritariamente composto por áreas alagadiças, de baixa valorização imobiliária e com grande concentração de aglomerados subnormais, uma forma de ocupação irregular do território, onde há carência de serviços públicos essenciais, altos índices de violência, precarização da vida e da sobrevivência humana ${ }^{7}$.

De acordo com Rosemari ${ }^{8}$, existe uma clara correlação entre a condição de saúde e vulnerabilidade do indivíduo com o ambiente em que convive. Dessa forma, morar em bairros periféricos representa a falta de acesso a serviços de saúde, convivência com baixas condições de saneamento, presença de doenças infecciosas agudas e a falta de controle de doenças crônicas. Além disso, há a presença de afecções sociais como a violência, pobreza e baixo nível de educação.

A pandemia da COVID-19 também demonstrou maiores efeitos com populações periféricas, como a do bairro do Telégrafo. Apesar da sintomatologia geral da doença ser leve, os indivíduos vulneráveis e pobres estão mais propensos a evoluir para quadros graves e óbitos, assim como, os seus territórios apresentam os maiores índices da propagação da doença ${ }^{9}$.

Nesse contexto, a Atenção Primária à Saúde se insere como o nível primário de atenção e também como estratégia de organização do sistema de saúde. A APS brasileira prevê uma assistência focada na família, com orientação comunitária e competência cultural. O foco na família consiste na consideração desta como uma unidade que precisa de cuidados e que está inserida em uma orientação comunitária, ou seja, faz parte de uma comunidade com costumes, culturas, singularidades e coletividades, vivendo em função de um contexto social, econômico e físico ${ }^{10}$.

Dessa forma, a Terapia Ocupacional, inserida na Atenção Primária, procura respeitar a comunidade em que atua, através de uma visão focada no sujeito em suas interfaces, demandas e contextos físicos, mentais, sociais e, sobretudo, ocupacionais. As ações, atribuições e tecnologias da Terapia Ocupacional na APS, assim como outras profissões, é pautada na intersetorialidade e no trabalho interdisciplinar, principalmente com advento e implantação dos Núcleos de Apoio à Saúde da Família, de 2008.

\section{Nesse contexto, a}

Atenção Primária à

Saúde se insere como

o nível primário de

atenção e também

como estratégia

de organização do

sistema de saúde. A

APS brasileira prevê

uma assistência

focada na família,

com orientação

comunitária e

competência

\section{cultural.}

Dentro do arcabouço teórico e prático da Terapia Ocupacional, a prevenção, proteção e promoção de saúde ocorrem através das atividades e da ocupação, apoiada pela relação do sujeito inserido em um ambiente biopsicossocial. Esse pensamento se assemelha com os princípios de orientação comunitária e competência cultural citados anteriormente, demonstrando a proximidade e a relação positiva que a categoria pode oferecer dentro do contexto de atenção primária ${ }^{10,11}$.

A experiência de atendimentos e visitas domiciliares ocorridas, se assemelham as vivências das autoras Baissi e Maxta ${ }^{12}$, que relatam os cuidados familiares proporcionados por uma UMS do município de Varzea Paulista. As autoras demonstram como a Terapia Ocupacional pode atuar nos atendimentos domiciliares, identificando necessidades das famílias, realizando o manejo correto das dificuldades ocupacionais apresentadas, a exemplo de utilização de técnicas de conservação de energia, adaptações ambientais e também organogramas familiares e ecomapas.

Quanto a utilização dos grupos terapêuticos, essa estratégia é citada na literatura como uma das propostas amplamente utilizadas por terapeutas ocupacionais e pela equipe na atenção primária. Os grupos terapêuticos apresentam aspectos positivos na promoção da saúde dos indivíduos, permitindo a ampliação de autonomia, sendo um dispositivo potencializador de criação de vínculos, mobilizador de questionamentos, transformador de relações com o espaço social e físico, bem como cumpre efetivamente um papel de promoção de educação popular e formação do sujeito ${ }^{13,14}$.

Os atendimentos e acompanhamentos em vigilância do desenvolvimento infantil também são utilizadas por terapeutas ocupacionais atuantes na APS. Seja por serviços complexos ou por atendimentos esporádicos, a valorização do acompanhamento do desenvolvimento infantil, desde a gestação até os primeiros anos de vida, é uma estratégia importante e recomendada para assegurar a saúde da criança e de seus cuidadores. A terapia ocupacional pode trabalhar aspectos globais, como cuidados na gestação, formação de vínculos afetivos saudáveis, calendário de vacinação, prevenção de acidentes e avaliação do desenvolvimento neuromotor, até coisas específicas como o engajamento da família no papel ocupacional de cuidados, engajamento da criança no brincar e estimulação precoce ${ }^{14}$.

Ações como as realizadas dentro da UMS Telégrafo, também são encontradas 


\section{artigo}

em outras produções acadêmicas, como destacam Cabral e Bregalda ${ }^{15}$, em uma revisão de literatura que buscou identificar a atuação do terapeuta ocupacional em Unidades Básicas de Saúde brasileiras. A presença de residentes, bem como atendimentos individuais a todas as faixas etárias, grupos terapêuticos, visitas domiciliares e vigilância do desenvolvimento, foram levantados pelas autoras em diferentes serviços da região Sudeste e Nordeste do Brasil.

\section{CONCLUSÃO}

De acordo com o que foi exposto, foi observado que o terapeuta ocupacional pode atuar na atenção primária em diferentes frentes. Apesar das possibilidades de ação da categoria, há a necessidade de realização de relatos de experiência, como a deste artigo, oportunizando outros profissionais a observar e analisar as vivências dos autores.

Durante o levantamento bibliográfico, foi observado que terapeutas ocupacionais de outros serviços de atenção primária também realizam ações diferentes das dos autores, como atendimentos mais específicos em atenção primária, confecção de tecnologia assistiva e atendimentos a usuários de álcool e drogas, bem como outras frentes e possibilidades. As produções encontradas não destacavam se o território era marcado pela fragilidade social, havendo essa sugestão de pesquisa para futuras produções. -

\section{REFERÊNCIAS}

1. Declaração de Alma-Ata. In: Conferência Internacional sobre Cuidados Primários de Saúde. 1978 Set 6-12; Alma Ata, Cazaquistão

2. Brasil. Ministério da Saúde. Secretaria de Atenção à Saúde. Departamento de Atenção Básica [Homepage da Internet]. Avaliação na Atenção Básica [acesso em 20 jan 2021]. Brasília, 2006. Disponível em: https://bvsms.saude.gov.br/bvs/publicacoes/manual_avaliacao_atencao_primaria.pdf

3. Conselho Regional de Fisioterapia e Terapia Ocupacional da $12^{\circ}$ Região (CREFITO-12) [Homepage da Internet]. Conceito da Terapia Ocupacional [acesso em 20 jan 2021]. Disponível em: https:// crefito12.org.br/terapia-ocupacional/

4. COFFITO. CONSELHO FEDERAL DE FISIOTERAPIA E TERAPIA OCUPACIONAL. Resolução n 407 de 18 de agosto de 2011a. Disciplina a Especialidade Profissional Terapia Ocupacional em Saúde da Família e dá outras providências. Diário Oficial da União [Internet]. 2011 Disponivel em: http://www.coffito.org.br/publicacoes/ pub_view.asp?cod=2137\&psecao $=9$

5. ESUS-AB [Homepage da Internet]. Ficha de Cadastro Domiciliar e Territorial. Ocupacional [acesso em 20 jan 2021]. Disponível em: https://integracao.esusab.ufsc.br/v211/docs/dicionario-fcd.html

6. Silva JM, Chagas CAN. A Dinâmica Da (Re) Produção Espacial E A Novas Territorialidades Em Belém-Pa: Disputa Pelo Poder E A Cartografia De Homicídios Na Área Da $7^{\text {a }}$ Aisp. In: Anais do VI Congreso Iberoamericano de Estudios Territoriales y Ambientales; 2014 Sept 8-12; São Paulo, Brasil: Universidade Estadual Paulista (UNESP); 2014; 2564. Disponivel em: https://www.researchgate.net/profile/clay_chagas/publication/274380206_a_dinamica_da_re_producao_espacial_e_a_novas_territorialidades_em_belempa_disputa_pelo_ poder_e_a_cartografia_de_homicidios_na_area_da_7a_aisp/ links/551d46d30cf2000f8f938b74/a-dinamica-da-re-producao-espacial-e-a-novas-territorialidades-em-belem-pa-disputa-pelo-poder-e-a-cartografia-de-homicidios-na-area-da-7aaisp.pdf

7. Instituto Brasileiro de Geografia e Estatística [Homepage da Internet]. Censo 2010 [acesso em 20 jan 2021]. Disponivel em: www.ibge.gov.br;

8. Pilati R; Sales IKP; Santos AA; Silva CA; Fidalgo Neto AA; Tubino
R; Saldanha RR. O Ambiente Como Fator De Risco A Saúde De Crianças De Bairro Periférico De Brasília-DF. Ensaios e Ciência: Ciências Biológicas, Agrárias e da Saúde [online], 2013 [Acesso em 13 Jan 2021]; 17(5): 51-62. Disponivel em: https://revista.pgsskroton. com/index.php/ensaioeciencia/article/view/2299

9. Estrela FM. Pandemia da Covid 19: refletindo as vulnerabilidades a luz do gênero, raça e classe. Ciência \& Saúde Coletiva [online]. 2020 [Acesso em 13 Jan 2021]; 25(9): 3431-3436. Disponível em: <https://doi.org/10.1590/141381232020259.14052020>. ISSN 1678-4561. https://doi. org/10.1590/1413-81232020259.14052020.

10. Mendes EV. A Construção Social Da Atenção Primária À Saúde. 1 ed. Brasília: Conselho Nacional de Secretários de Saúde - CONASS, 2015. $193 \mathrm{p}$.

11. Rocha EF, Paiva LFA, Oliveira RH. Terapia ocupacional na Atenção Primária à Saúde: atribuições, ações e tecnologias. Cad. Bras. Ter. Ocup [online]. 2012 [Acesso em 13 Jan 2021]; 20(3):351361. Disponivel em: http://www.cadernosdeterapiaocupacional. ufscar.br/index.php/cadernos/article/view/679

12. Baissi G.; Maxta BSB. Experiência da Terapia Ocupacional no cuidado familiar em um serviço de Atenção Primária em Saúde. Cad. Ter. Ocup [online]. 2013 [Acesso em 13 Jan 2021]; 21(1): 413422. Disponivel em: http://www.cadernosdeterapiaocupacional. ufscar.br/index.php/cadernos/article/view/828

13. Minozzo F, et al. Grupos de saúde mental na atenção primária à saúde. Revista de Psicologia [online], 2012 [Acesso em 21 Jan 2021]; 24(2): 323-340. Disponivel em:https://www.scielo.br/scielo.php?script=sci_arttext\&pid=S1984-02922012000200008\&l$\mathrm{ng}=$ pt \&tlng=pt.

14. Lancman S; Barros JO. Estratégia de saúde da família (ESF), Núcleo de Apoio à Saúde da Família (NASF) e terapia ocupacional: problematizando as interfaces. Rev. Ter. Ocup. Univ [online]. 2011 [Acesso em 21 Jan 2021]; 22(3): 263-269. Disponível em: https:// doi.org/10.11606/issn.2238-6149.v22i3p263-269.

15. Cabral LRS; Bregalda MM. A atuação da terapia ocupacional na atenção básica à saúde: uma revisão de literatura. Cadernos de Terapia Ocupacional da UFSCar [online]. 2017 [Acesso em 08 Fev 2021]; 25(1): 179-189. Disponível em: http://dx.doi. org/10.4322/0104-4931.ctoAR0763. 